\title{
On-line Identification of Minor Flavones from Sugarcane Juice by LC/UV/MS and Post-Column Derivatization
}

\author{
Renata Colombo, ${ }^{a}$ Janete H. Yariwake, ${ }^{*, a}$ Emerson F. Queiroz, ${ }^{b}$ Karine Ndjoko $^{b}$ and Kurt Hostettmann ${ }^{b}$ \\ ${ }^{a}$ Instituto de Química de São Carlos, Universidade de São Paulo, CP 780, 13560-970 São Carlos-SP, Brazil \\ ${ }^{b}$ Laboratoire de Pharmacognosie et Phytochimie, École de Pharmacie Genève-Lausanne, \\ Université de Genève, Quai Ernest Ansermet 30, 1211, Genève 4, Genève, Switzerland
}

\begin{abstract}
Este trabalho apresenta a identificação "on line" de flavonas minoritárias do suco da cana-deaçúcar (Saccharum officinarum), por cromatografia líquida de alta eficiência com detector UV acoplada à espectrometria de massas (CL/UV/EM) com ionização química à pressão atmosférica, dissociação induzida por colisão (IQPA-DIC-EM/EM) e derivatização pós-coluna utilizando reagentes de deslocamento de UV. As análises CLAE-UV com reagentes de deslocamento forneceram informações sobre a posição da substituição no esqueleto dos flavonóides e, em combinação com dados de EM, estas técnicas permitiram a identificação "on-line" de cinco flavonas da garapa: luteolina-8- $C$-glucosil-7-O-glucuronídeo; tricina-7-O-neoesperosideo-4'$O$-ramnosídeo; tricina-7- $O$-metilglucuronato-4'-O-ramnosídeo; tricina-7-O-metilglucuronídeo; swertisina; e outras quatro substâncias foram parcialmente identificadas como flavonas glicosiladas. Somente a swertisina (7-O-metilapigenina-6- $C$-glicosídeo) foi anteriormente descrita no bagaço da cana-de-açúcar.
\end{abstract}

This work describes the on-line characterization of minor flavones from sugarcane (Saccharum officinarum) juice by high-performance liquid chromatography coupled to diode array UV detection and mass spectrometry (LC/UV/MS) using atmospheric pressure chemical ionizationcollision-induced dissociation (APCI-CID-MS/MS) and post-column derivatization using UV shift reagents. HPLC-UV analysis with shift reagents provided information about the substitution pattern in the flavonoid skeleton and, combined with MS data, these techniques allowed for the on-line identification of five "garapa" flavones: luteolin-8- $C$-glucosyl-7- $O$-glucuronide; tricin-7- $O$-neohesperoside-4'- $O$-rhamnoside; tricin-7- $O$-methylglucuronate-4'- $O$-rhamnoside; tricin-7-O-methylglucuronide; swertisin, while four other compounds were partially identified as glycosylflavones. Only swertisin (7- $O$-methylapigenin-6- $\mathrm{C}$-glucoside) was reported previously in sugarcane molasses.

Keywords: sugarcane juice, Saccharum officinarum L. (Poaceae), flavones, LC/UV/MS, UV shift reagents

\section{Introduction}

Hyphenated techniques such as liquid chromatography with UV photodiode array detection (HPLC-UV) and on-line liquid chromatography-ultraviolet detection-mass spectrometry (HPLC-UV-MS) are powerful techniques for the structural identification of compounds found in extracts prepared from very small amounts of plant material. In the case of plant polyphenolic compounds such as flavonoids and xanthones, the possibility of obtaining on-line UV data by a combination with the post-column addition of UV shift reagents is a very important step to validate the structure

*e-mail: janete@iqsc.usp.br assignments proposed for the position of oxygenated groups on the polyphenolic skeleton. ${ }^{1}$ Recently, HPLC-UV-MS and post-column UV-derivatization were employed in the study of Trifolium extracts containing clovamides, flavonoids and isoflavonoids and their UV data were discussed in detail. ${ }^{2}$

Hyphenated techniques may be the technique of choice in studies requiring analyses of several plant samples, as in metabolomic studies. ${ }^{1}$ Strategies such as metabolite profiling and metabolomic analysis ${ }^{3}$ require appropriate analytical methods for the precise and reproducible determination of the plant metabolites that usually occur in complex mixtures containing hundreds of compounds and that are sometimes obtained from very small amounts of plant material (e.g., from test-tube cultures). Besides the study of important 
biological functions, metabolomic analysis has a great potential for application in the assessment of safety and biological equivalence of genetically modified organisms, including transgenic plants.

Several flavonoids from Saccharum species were reported in a previous literature review. ${ }^{4}$ More recently, sugarcane cultivated commercially in Brazil (Saccharum officinarum L.) is under systematic investigation due to the potential role of this plant as a dietary source of flavonoids with antioxidant properties. ${ }^{5,6}$ Our investigation into the flavonoid composition of Brazilian sugarcane also takes into account recent studies suggesting the potential role of this plant as a dietary source of antioxidant flavonoids due to the in vivo antioxidant activity of a sugarcane phenolic extract, ${ }^{7}$ since the consumption of sugarcane juice as an "energetic drink" thanks to its high sugar content is very popular in Brazil. Moreover, the increasing importance of sugarcane for the production of 'green' fuels, in addition to its traditional use in tropical countries as the most important raw material for the production of sugar and byproducts, highlights the need to develop new commercial varieties with higher sugar content and greater resistance to Brazil's major sugarcane pest, the sugarcane borer Diatraea saccharalis Fabricius (Lepidoptera: Crambidae). In this context, transgenic sugarcane is being studied ${ }^{8}$ and preliminary results of other studies of genetically modified sugarcane plants have indicated the relevance of the minor components in chemometric analyses of sugarcane HPLC chromatograms. ${ }^{9}$ Therefore, this article reports the identification of additional minor flavones from sugarcane juice by HPLC-UV-MS techniques.

\section{Experimental}

\section{Reagents and materials}

HPLC-grade acetonitrile was obtained from Romil (Cambridge, UK). Deionized water was prepared using a Reinstwasser-System Clear Cartridge System (SG, Hamburg, Germany). Both the solvents were passed through Millipore filters (water: $0.45 \mu \mathrm{m} \mathrm{HA}$; acetonitrile: $0.50 \mu \mathrm{m} \mathrm{FH}$; Bedford, MA, USA). Analysis-grade formic acid was purchased from Fluka (Buchs, Switzerland). Sodium hydroxide, aluminium chloride and sodium acetate were purchased from SigmaAldrich Chemie (Steinheim, Germany).

\section{Preparation of samples}

Samples of sugarcane juice, obtained by crushing cleaned and peeled sugarcane stems, were purchased from various suppliers in São Carlos, SP, Brazil. Juice samples were transferred to plastic bottles and frozen at $c a .-10{ }^{\circ} \mathrm{C}$ for storage: immediately prior to sample preparation, the samples were thawed and homogenized. Sugarcane juice samples $(10 \mathrm{~mL})$ were sonicated with $10 \mathrm{~mL}$ of methanol for $1.5 \mathrm{~min}$ at room temperature, the extract was filtered, mixed with $2.0 \mathrm{~mL}$ water, reduced in volume to $2 \mathrm{~mL}$ on a rotary evaporator and purified by solid-phase extraction (SPE) using Oasis HLB cartridges ( 3 cc, $60 \mathrm{mg}$; $30 \mu \mathrm{m}$ particle size; Waters, Milford, MA, USA) pre-conditioned with $1 \mathrm{~mL}$ of methanol and $1 \mathrm{~mL}$ of water. The interfering compounds were eluted with $3 \mathrm{~mL}$ of water, whilst the flavonoids were obtained by elution with $3 \mathrm{~mL}$ of methanol. Purified flavonoid extracts were filtered through $0.5 \mu \mathrm{m}$ Fluorpore membranes (Millipore) prior to injection into the HPLC system.

\section{HPLC-UV/PAD analysis}

Analyses were performed using a Hewlett-Packard 1100 (Waldbronn, Germany) photodiode array detector (PAD) liquid chromatography system with a Waters Symmetry $\mathrm{C}_{18}$ column $(250 \mathrm{~mm} \times 4.6 \mathrm{~mm}$ i.d.; $5 \mu \mathrm{m})$ protected by a SymmetryShield $\mathrm{C}_{18}$ guard column $(20 \times 3.9 \mathrm{~mm}$ i.d.; $5 \mu \mathrm{m})$. The mobile phase consisted of $0.2 \%$ formic acid in water (solvent A) and acetonitrile (solvent B). The elution program was: 0-8 min, linear gradient from 10 to $13 \% \mathrm{~B}$; 8-25 min, linear gradient from 13 to $20 \% \mathrm{~B} ; 25-40 \mathrm{~min}$, linear gradient from 20 to $40 \% \mathrm{~B}$; and 40-45 min, linear gradient from $40-60 \% \mathrm{~B}$. The flow rate was $1.2 \mathrm{~mL} \mathrm{~min}^{-1}$ and the sample injection volume was $10 \mu \mathrm{L}$. UV spectra were acquired between 200 to $600 \mathrm{~nm}$.

\section{HPLC-MS analysis}

Analysis were performed using a Finnigan MAT LCQ (San Jose, CA, USA) ion trap mass spectrometer equipped with an atmospheric pressure chemical ionization (APCI) interface operated under the following conditions: positive ion mode; capillary voltage, $11 \mathrm{~V}$; capillary temperature, $150{ }^{\circ} \mathrm{C}$; source voltage, $5 \mathrm{kV}$; vaporizer temperature, $450{ }^{\circ} \mathrm{C}$; corona needle current, $5 \mu \mathrm{A}$; sheath gas, nitrogen at $60 \mathrm{psi}$. Analyses were made in the full scan mode (150-800 u). The CID-MS/MS analyses were also made in a Finnigan MAT LCQ using helium as collision gas, and collision energy was set at $40 \mathrm{eV}$.

HPLC-UV/PAD analysis with post-column addition of shift reagents

The reagents used in the post-column derivatization system were: aqueous sodium hydroxide $\left(0.01 \mathrm{~mol} \mathrm{~L}^{-1}\right)$; 
aqueous aluminium chloride $\left(0.3 \mathrm{~mol} \mathrm{~L}^{-1}\right)$ and aqueous sodium acetate $\left(0.5 \mathrm{~mol} \mathrm{~L}^{-1}\right)$. The reaction was performed at room temperature except with aluminium chloride where the reaction coil was heated to $60{ }^{\circ} \mathrm{C}$. The classical shift reagents were prepared according to the literature. ${ }^{10}$ The solvent delivery system comprised two M-6000 pumps, a M-720 gradient controller and a U6K injector (Waters). The photodiode array detector HP-1040A (Hewlett-Packard) coupled with an HP-85 personal computer (HewlettPackard) was used for recording chromatograms and UV-Vis spectra. For post-column derivatization, an Eldex Model A-30-5-2 (Eldex Labs., Menlo Park, CA, USA) pump and a reaction coil were employed. Shift reagents were added to the eluent at a flow rate of $0.3 \mathrm{~mL} \mathrm{~min}^{-1}$.

\section{Results and Discussion}

Figure 1 depicts a typical chromatogram obtained from sugarcane juice, identifying the marked peaks. The compounds corresponding to peaks 1 (schaftoside), 2 (isoschaftoside), 5 (vitexin), 9 (4',5'-dimethyl-luteolin8-C-glycoside), 11 (tricin-7- $O$-neohesperoside), and
16 (tricin-7-O-glycoside) were identified and reported in one of our previous studies of sugarcane juice. ${ }^{11}$ On the other hand, although the compounds corresponding to peaks 3 (diosmetin-8- $C$-glycoside), 12 (tricin-7- $O$ rhamnosylgalacturonide), 15 (tricin-4'-O-(erythro- or threo-guaiacylglyceryl) ether-7-O-glucopyranoside), and 19 (tricin-4'-O-(erythro- or threo-guaiacylglyceryl) ether) were also reported previously, they had been found only in sugarcane leaves or bagasse. ${ }^{12}$ The structural elucidation of the five additional minor flavones from sugarcane juice (Figure 2) is discussed below, and the HPLC-APCI-CID-MS and on-line UV data of these compounds are shown in Table 1. Some of the identifications and discussions are also based on the comparison of data herein shown with those obtained in a study using exact mass measurement for unequivocal elucidation of isomeric sugarcane flavones. ${ }^{13}$

The APCI spectrum of compound $\mathbf{4}$ presented an $[\mathrm{M}+\mathrm{H}]^{+}$ion at $\mathrm{m} / \mathrm{z} 625$ and an abundant fragment at $\mathrm{m} / \mathrm{z}$ $449[\mathrm{M}+\mathrm{H}-176]^{+}$. The loss of $176 \mathrm{u}$ is characteristic of a glucuronic acid moiety, ${ }^{14,15}$ while the ion at $m / z, 449$ suggests a luteolin- $C$-glucoside derivative. CID-MS/MS of ion $m / z, 449$ generated fragments at $m / z, 431 ; 413 ; 395$;

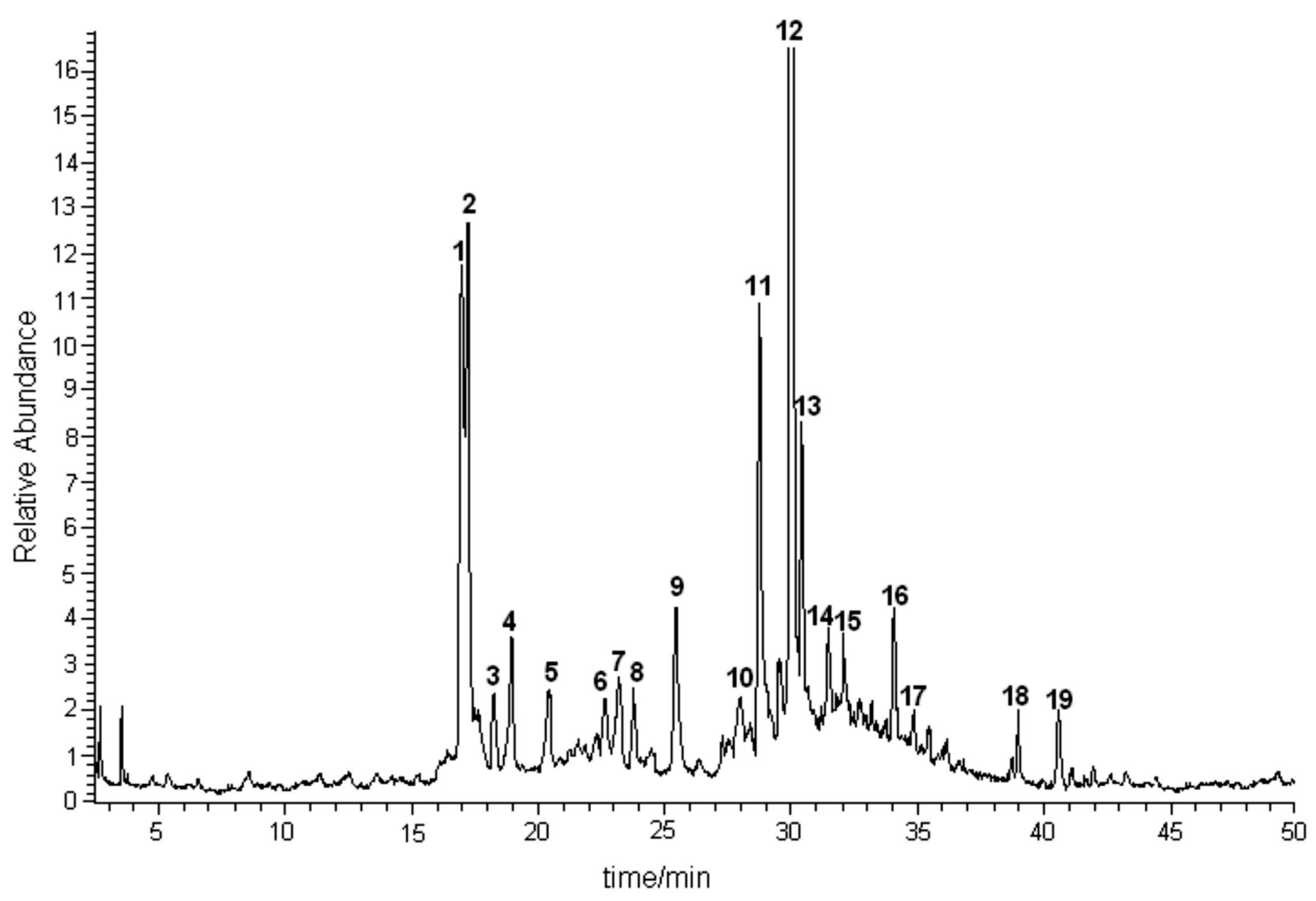

Figure 1. TIC-LC/APCI-MS of sugarcane juice extract. The chromatographic and MS conditions are described in the Experimental section. Peaks identification: $\mathbf{1}$ (schaftoside), $\mathbf{2}$ (isoschaftoside), $\mathbf{3}$ (diosmetin-8- $C$-glycoside), $\mathbf{4}$ (luteolin-8- $C$-glucosyl-7- $O$-glucuronide), $\mathbf{5}$ (vitexin), 8 (swertisin), 9 (4',5'-dimethylluteolin-8- $C$-glycoside), 10 (tricin-7- $O$-neohesperoside-4'- $O$-rhamnoside), 11 (tricin-7- $O$-neohesperoside), 12 (tricin-7- $O$ rhamnosylgalacturonide), 15 (tricin-4'-O-(erythro- or threo-guaiacylglyceryl) ether-7- $O$-glucopyranoside), 16 (tricin-7- $O$-glycoside), 17 (tricin-7- $O$ methylglucuronate-4'-O-rhamnoside), 18 (tricin-7-O-methylglucuronide), 19 (tricin-4'-O-(erythro- or threo-guaiacylglyceryl) ether). Peaks 6, 7, 13 and $\mathbf{1 4}$ are flavones not fully identified (see also Results and Discussion). 
$383 ; 353,329,311$ and 299, which are characteristic of the $C$-glucoside fragmentation pathway (Figure 2) and are identical to those described for luteolin-8-C-glucoside (orientin). ${ }^{16}$ The position of the glucuronic acid and glucose moieties was also consistent with the data from the UV

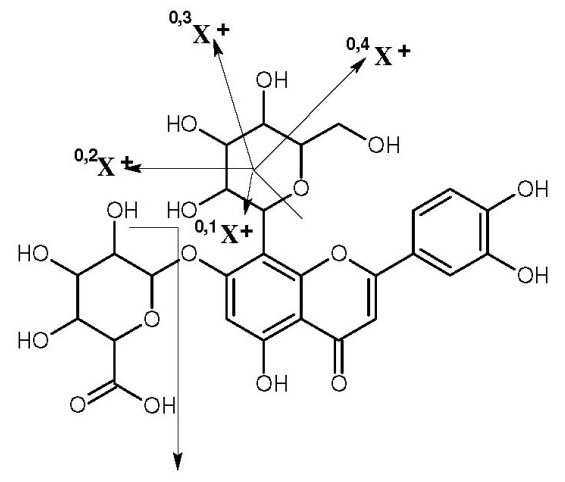

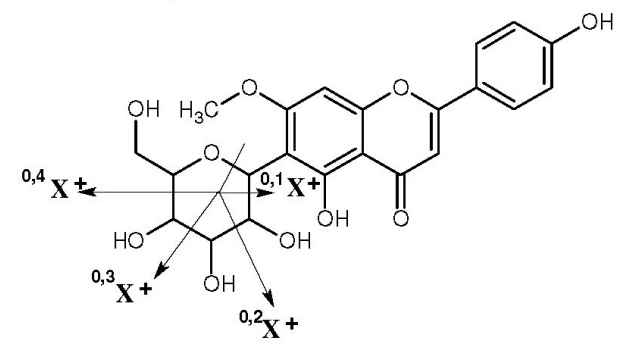<smiles>CCC(C)(Oc1cc(O)c2c(=O)cc(-c3cc(OC)c(OCC(C)(CC)c4ccccc4)c(OC)c3)oc2c1)[GeH2]c1ccccc1</smiles><smiles></smiles><smiles>CCC(C)(C)Oc1cc(O)c2c(=O)cc(-c3cc(OC)c(O)c(OC)c3)oc2c1</smiles>

analysis with post-column addition of shift reagents: the addition of sodium acetate showed a shoulder in band I, indicating a free $\mathrm{OH}$ group at 4', while the absence of shift to band II indicated a substituted 7-OH group. On the other hand, the addition of aluminum chloride led to a shift of

4, Luteolin-8-C-glucosyl-7-O-glucuronide

$[\mathrm{M}+\mathrm{H}]^{+}: 625$

Glu: $-176 \mathrm{u}$

${ }^{0,1} \mathrm{X}^{+}:-150 \mathrm{u}$

${ }^{0,2} \mathrm{X}^{+}:-120 \mathrm{u}$

${ }^{0,3} \mathrm{X}^{+}:-90 \mathrm{u}$

${ }^{0,4} \mathrm{X}^{+}:-60 \mathrm{u}$

8, Swertisin (7-O-methylapigenin-6- $\mathrm{C}$ glucoside)

$[\mathrm{M}+\mathrm{H}]^{+}: 447$

${ }^{0,1} \mathrm{X}^{+}:-150 \mathrm{u}$

${ }^{0,2} \mathrm{X}^{+}:-120 \mathrm{u}$

${ }^{0,3} \mathrm{X}^{+}:-90 \mathrm{u}$

${ }^{0,4} X^{+}:-60 u$

10, Tricin-7- $O$-neohesperoside-4'- $O$ rhamnoside

$[\mathrm{M}+\mathrm{H}]^{+}: 785$

Rha: $-146 \mathrm{u}$

Rha-Glc: $-308 \mathrm{u}$

17, Tricin-7-O-methylglucuronate-4'-Orhamnoside

$[\mathrm{M}+\mathrm{H}]^{+}: 667$

Rha: $-146 \mathrm{u}$

MeGlu: $-190 \mathrm{u}$

18, Tricin-7- $O$-methylglucuronide

$[\mathrm{M}+\mathrm{H}]^{+}: 521$

MeGlu: -190 u

Figure 2. Structure of the flavones from sugarcane juice described in this work and fragmentation pathways. Numbering of the compounds as in Figure 1. Fragments nomenclature as in reference 16. Glu = glucuronic acid; Glc = glucose; Rha = rhamnose; MeGlu = methylglucuronide . 
Table 1. On-line HPLC-APCI-CID-MS and UV/DAD data of sugarcane juice flavones described in this work. Numbering according to Figure 1

\begin{tabular}{|c|c|c|c|c|c|c|c|c|}
\hline & \multirow{2}{*}{\multicolumn{2}{|c|}{$[\mathrm{M}+\mathrm{H}]^{+}$CID-MS fragment ions $(\mathrm{m} / \mathrm{z}$, relative intensity) }} & \multicolumn{2}{|c|}{ UV spectra / nm } & \multicolumn{2}{|c|}{ UV spectra NaOAc / nm } & \multicolumn{2}{|c|}{ UV spectra $\mathrm{AlCl}_{3} / \mathrm{nm}$} \\
\hline & & & Band I & Band II & Band I & Band II & Band I & Band II \\
\hline 4 & 625 & $\begin{array}{l}449(4), 431(100), 413(35), 395(8), 383(28), 353 \\
(11), 329(18), 311(3), 299(2)\end{array}$ & 330 & 275 & $330,395 \mathrm{sh}$ & 275 & 400 & 245 \\
\hline *6 & 617 & $401(100), 371(27), 331(4)$ & 321 & 282 & 322 & 282 & 388 & 282 \\
\hline$* 7$ & 617 & 419 (100), $387(50), 327$ (6) & 327 & 268 & 327 & 268 & 327 & 268 \\
\hline 8 & 447 & $\begin{array}{l}429(100), 411(31), 393(18), 381(66), 351(36), 327 \\
(12), 297(8)\end{array}$ & 343 & 265 & $349,397 \mathrm{sh}$ & 265 & 402 & 265 \\
\hline 10 & 785 & $639(100), 493(7), 331(52)$ & 344 & 269 & 344 & 269 & 395 & 269 \\
\hline$* 13$ & 659 & 497 (37), 315 (100) & 330 & 271 & 330 & 271 & 389 & 271 \\
\hline$* 14$ & 571 & $463(72), 331(100)$ & 323 & 273 & 323 & 273 & 374 & 273 \\
\hline 17 & 667 & $521(26), 331(100)$ & 323 & 279 & 323 & 279 & 375 & 279 \\
\hline 18 & 521 & $331(100)$ & 333 & 272 & $333,392 \mathrm{sh}$ & 272 & 392 & 272 \\
\hline
\end{tabular}

sh: shoulder; *flavones not fully identified (see also Results and Discussion).

$71 \mathrm{~nm}$, indicating a free $5-\mathrm{OH}$ group and the presence of the ortho-dihydroxyl group. Therefore, the glucuronic acid moiety was attributed to the 7- position, also considering that CID-MS/MS data and ${ }^{0,2} \mathrm{X}$ ion ${ }^{16}$ (Figure 2) pointed to an 8-C-glucoside.

The compound $\mathbf{8}$ was identified as being swertisin (7-O-methylapigenin-6- $C$-glucoside), reported previously in sugarcane molasses and also in other plant sources. ${ }^{4,14}$ This proposition is confirmed by the $[\mathrm{M}+\mathrm{H}]^{+}$ion at $\mathrm{m} / \mathrm{z}$ 447 and by the peak at $\mathrm{m} / \mathrm{z} 327$, which indicates a loss of $120 \mathrm{u}\left({ }^{0,2} \mathrm{X}\right.$ ion, ${ }^{16}$ Figure 2$)$ from the $[\mathrm{M}+\mathrm{H}]^{+}$ion, a characteristic feature of a $C$-glucoside flavonoid. ${ }^{16}$ The CID-MS/MS data also indicated a fragmentation pathway of a $C$-linked glucose moiety (Figure $2 ; m / z, 429,411$, $393,381,351,327$ and 297), and analysis of ${ }^{0,2} \mathrm{X}$ ion indicated an 6-C-glucoside. ${ }^{16}$ The UV analysis with postcolumn addition of sodium acetate showed the absence of a bathochromic shift of band I, confirming the presence of a blocked 7-OH group and showed also a shoulder in band II indicating a free $\mathrm{OH}$ group in position 4'. The UV spectrum obtained with aluminum chloride, while confirming a free 5-OH group, also reveals the absence of ortho-dihydroxyl groups.

For compound 10, identified as tricin-7-O-neohesperoside-4'-O-rhamnoside, the MS and CID-MS/MS analyses showed respectively the $[\mathrm{M}+\mathrm{H}]^{+}$ion at $m / z, 785$ and the fragments at $m / z 639,493$ and 331. These fragments have already been described for tricin 7-O-neohesperidoside (compound 11) ${ }^{11}$ and were also studied by exact mass measurement. ${ }^{13}$ The fragment at $\mathrm{m} / \mathrm{z}, 639[\mathrm{M}+\mathrm{H}-146]^{+}$ indicated loss of a rhamnose moiety. ${ }^{14,17}$ The UV data obtained with sodium acetate showed positions 7, 3' and 4' blocked, while the 5-OH group was free.

In the case of compound 17, the peak at $\mathrm{m} / \mathrm{z}, 667$ corresponded to the $[\mathrm{M}+\mathrm{H}]^{+}$ion. The CID-MS/MS fragments at $\mathrm{m} / \mathrm{z} 521$ and 331 (the latter indicating the aglycone $\operatorname{tricin}^{11,12}$ ), showed a loss of 146 and $190 \mathrm{u}$, respectively. The loss of $146 \mathrm{u}$ is characteristic of a rhamnose moiety, ${ }^{14,17}$ while the loss of $190 \mathrm{u}$ is reported in the literature as indicative of a methylglucoronic acid group. ${ }^{18,19}$ The identification of tricin-7- $O$-methylglucuronate-4'- $O$-rhamnoside was also confirmed by the absence of a bathochromic shift in band II with addition of sodium acetate, indicating that position 7 was blocked by the methylglucuronic acid moiety. The absence of a bathochromic shift and shoulder in band I pursuant to the addition of sodium acetate also indicated that positions 3' and 4' were blocked.

APCI-MS data of compound $\mathbf{1 8}$ showed the $[\mathrm{M}+\mathrm{H}]^{+}$ion located at $m / z 521$, while the ion at $m / z, 331$ was attributed to the $[\mathrm{A}+\mathrm{H}]^{+}$ion of tricin. In addition, the loss of $190 \mathrm{u}$ was attributed to a methylglucuronic acid moiety (as in 17). The UV analysis with shift reagents indicated the free 4' position, due to the presence of a shoulder in band I resulting from the addition of sodium acetate, while the absence of a shift in band II indicated a substituted 7-OH group.

The identity of the compounds corresponding to peaks 6, 7, 13 and $\mathbf{1 4}$ was not fully elucidated, but some partial propositions about their structure were deduced from their HPLC-UV-MS data (Table 1). All these compounds are glycosilated flavones but the full identification of the nature and position of the substituent groups was not achieved.

\section{Conclusions}

Among the five compounds fully described here, only swertisin was previously reported in sugarcane molasses. ${ }^{4}$ Another triglycosilated flavonoid from sugarcane leaves 
is described in the literature, ${ }^{4}$ but this is the first report of the triglycosilated flavone $\mathbf{1 0}$ in sugarcane. Three other compounds were partially identified as tricin derivatives. Several tricin derivatives have already been described in Saccharum species. ${ }^{5,11,12,20}$

The hyphenated HPLC-UV-MS technique proved to be a very important tool for the on-line recording of structural information about minor compounds in a complex matrix such as that of sugarcane juice. Therefore, the on-line structural elucidation of components can offer valuable contributions to the scope of the analytical chemistry (scientific studies or technological applications such as quality assessment) of extracts of plant foods such as sugarcane and its derivatives.

\section{Acknowledgments}

The authors wish to thank the Brazilian institutions FAPESP (fellowship to R.C., 00/11645-2; research grant to J. H. Y., 06/59457-6) and CNPq (fellowship to J. H. Y.) for their financial support, and the Post-Graduate Dean's Office of the Universidade de São Paulo (travel fellowship to R. C.). Swiss financial support for this work was provided by the Swiss National Science Foundation and the Fondation Herbette, University of Lausanne.

\section{Supplementary Information}

Supplementary data are available free of charge at http://jbcs.sbq.org.br as PDF file.

\section{References}

1. Wolfender, J.-L.; Ndjoko, K.; Hostettmann, K.; J. Chromatogr., A 2003, 1000, 437 and references cited herein.

2. Polasek, J.; Queiroz, E. F.; Hostettmann, K.; Phytochem. Anal. 2007, 18, 13.

3. Fiehn, O.; Plant Mol. Biol. 2002, 48, 155.
4. Smith, P.; Paton, N. H.; Sugar Technol. Rev. 1985, 12, 117.

5. Vila, F. C.; Colombo, R.; de Lira, T. O.; Yariwake, J. H.; J. Braz. Chem. Soc. 2008, 19, 903 and references cited herein.

6. Ferreira, R. Q.; de Lira, T. O.; Zeraik, M. L.; Yariwake, J. H.; Avaca, L. A.; J. Agric. Food Chem., submitted.

7. Duarte-Almeida, J. M.; Novoa, A. V.; Linares, A. F.; Lajolo, F. M.; Genovese, M.; Plant Food Hum. Nutr. 2006, 61, 187.

8. Falco, M. C.; Silva-Filho, M. C.; Plant Physiol. Biochem. 2003, 41,761 .

9. de Lira, T. O.; Yariwake, J. H.; Ribeiro, J.; Ferreira, M. M. C., unpublished work.

10. Ducrey, B.; Wolfender, J.-L.; Marston, A.; Hostettmann, K.; Phytochemistry 1995, 38, 129.

11. Colombo, R.; Yariwake, J. H.; Queiroz, E. F.; Ndjoko, K.; Hostettmann, K.; Phytochem. Anal. 2006, 17, 337.

12. Colombo, R.; Yariwake, J. H.; Queiroz, E. F.; Ndjoko, K.; Hostettmann, K.; J. Chromatogr., A 2005, 1082, 51.

13. Colombo, R.; Yariwake, J. H.; McCullagh, M.; J. Braz. Chem. Soc. 2008, 19, 483.

14. Wolfender, J.-L.; Rodriguez, S.; Hostettmann, K.; J. Chromatogr., A 1998, 794, 299.

15. Schütz, K.; Kammerer, D.; Carle, R.; Schieber, A.; J. Agric. Food Chem. 2004, 52, 4090.

16. Waridel, P.; Wolfender, J.-L.; Ndjoko, K.; Hobby, K. R.; Major, H. J.; Hostettmann, K.; J. Chromatogr., A 2001, 926, 29 and references cited herein.

17. de Rijke, E.; Out, P.; Niessen, W. M. A.; Ariese, F.; Gooijer, C.; Brinkman, U. A. T.; J. Chromatogr., A 2006, 1112, 31.

18. Souleles, C.; J. Nat. Prod. 1989, 52, 1311.

19. Huellin, J.; Lin, Y. T.; Huang, Y. J.; Wen, K. C.; Chen, R. M.; Ueng, T. H.; Liao, C. H.; J. Food Drug Anal. 2001, 9, 6.

20. Duarte-Almeida, J. M.; Negri, G.; Salatino, A.; de Carvalho, J. E.; Lajolo, F. M.; Phytochemistry 2007, 68, 1165.

Received: October 22, 2008

Web Release Date: August 31, 2009

FAPESP helped in meeting the publication costs of this article. 


\section{On-line Identification of Minor Flavones from Sugarcane Juice by LC/UV/MS and Post-Column Derivatization}

\section{Renata Colombo, ${ }^{a}$ Janete H. Yariwake, ${ }^{*, a}$ Emerson F. Queiroz, ${ }^{b}$ Karine Ndjoko ${ }^{b}$ and Kurt Hostettmann ${ }^{b}$}

${ }^{a}$ Instituto de Química de São Carlos, Universidade de São Paulo, CP 780, 13560-970 São Carlos-SP, Brazil

${ }^{b}$ Laboratoire de Pharmacognosie et Phytochimie, École de Pharmacie Genève-Lausanne, Université de Genève, Quai Ernest Ansermet 30, 1211, Genève 4, Genève, Switzerland

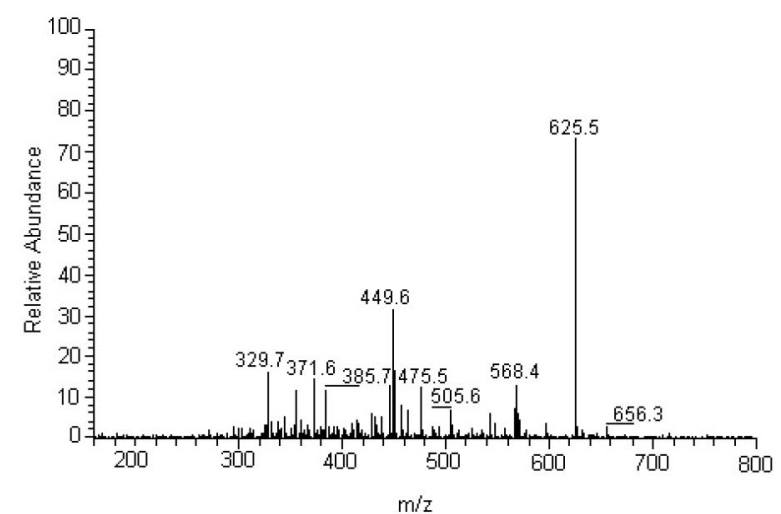

(a)

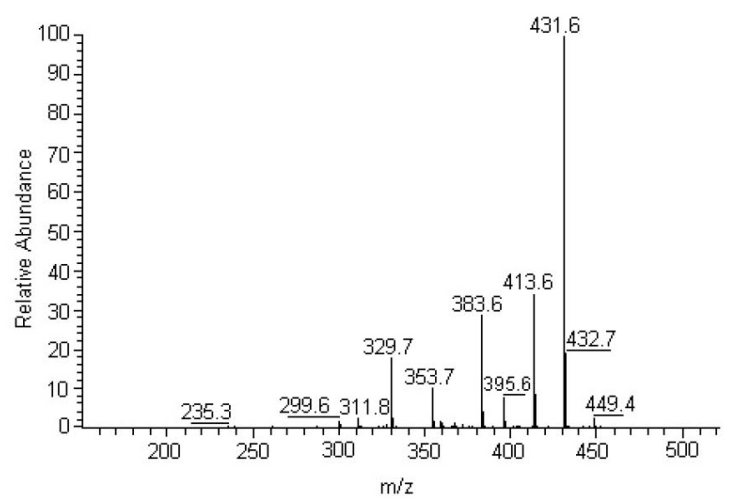

(b)

Figure S1. HPLC-APCI-CID-MS/MS spectra of compound 4. (a) CID-MS; (b) CID-MS/MS of ion $m / z 449$.

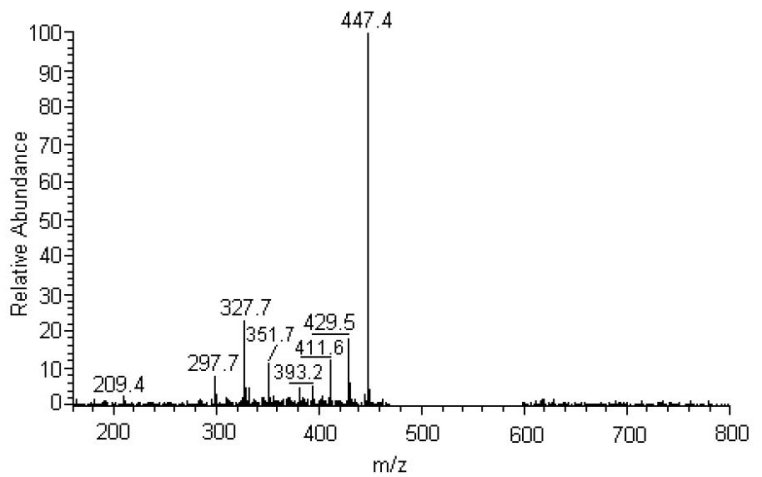

(a)

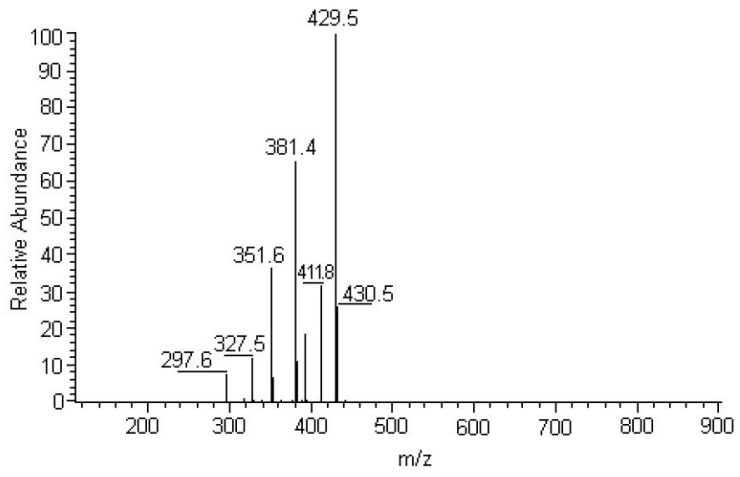

(b)

Figure S2. HPLC-APCI-CID-MS spectra of compound 8. (a) CID-MS; (b) CID-MS/MS of ion $m / z 447$. 


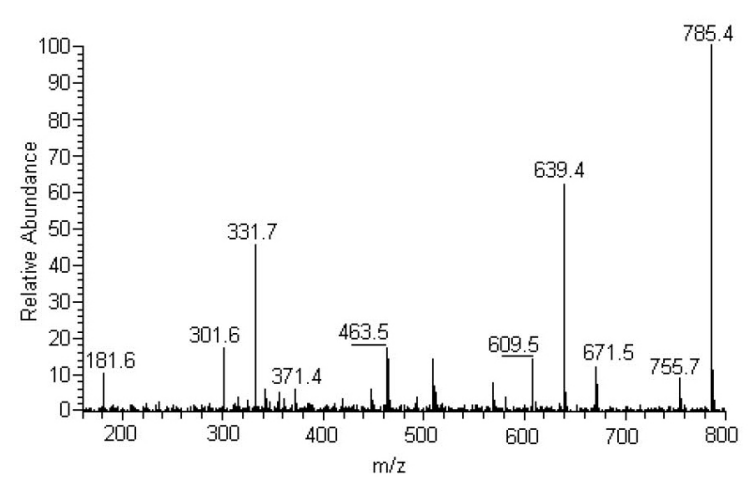

(a)

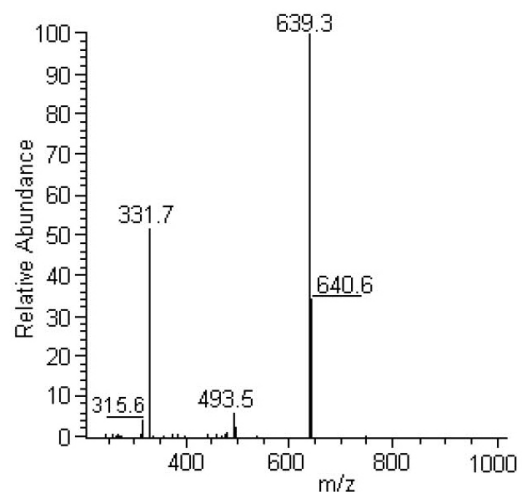

(b)

Figure S3. HPLC-APCI-CID-MS spectra of compound 10. (a) CID-MS; (b) CID-MS/MS of ion $m / z, 785$.

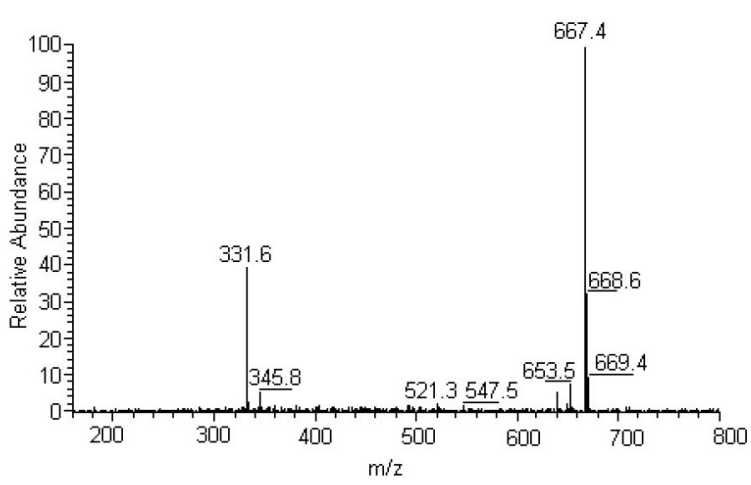

(a)

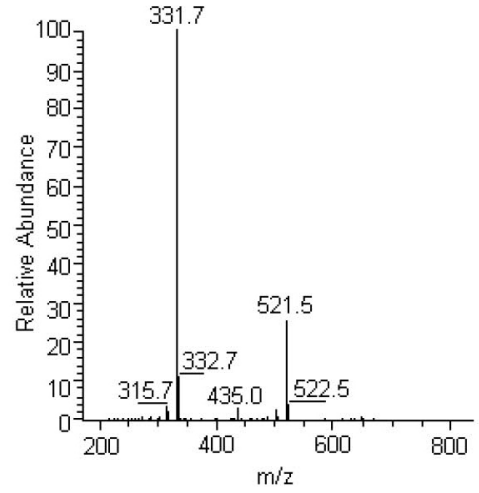

(b)

Figure S4. HPLC-APCI-CID-MS spectra of compound 17. (a) CID-MS; (b) CID-MS/MS of ion $m / z, 667$.

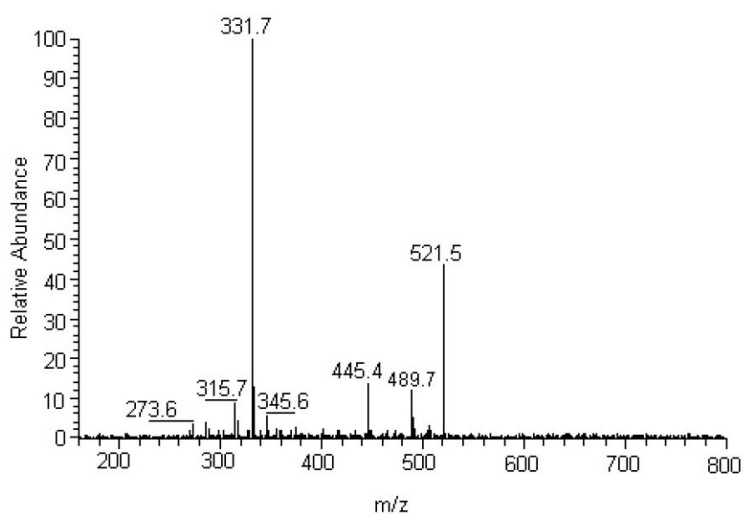

(a)

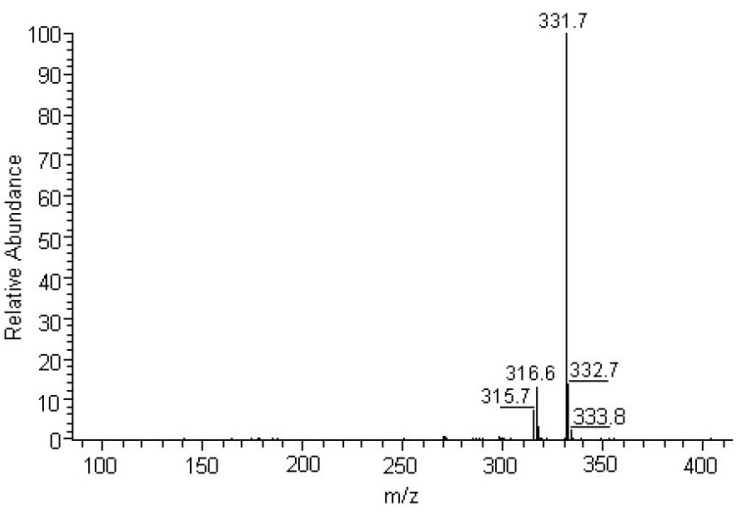

(b)

Figure S5. HPLC-APCI-CID-MS spectra of compound 18. (a) CID-MS; (b) CID-MS/MS of ion $m / z 521$. 